\title{
ON CHARACTERIZING DERIVATIVES
}

\author{
D. PREISS AND M. TARTAGLIA
}

(Communicated by Andrew M. Bruckner)

\begin{abstract}
We show that the set of derivatives of real functions of a real variable can be characterized by prescribing, for each subset of the real line, the set of its possible inverse images.
\end{abstract}

For a long time various authors have studied problems whose solutions would bring us closer to a characterization of those functions which are derivatives of a real-valued function of a real variable. The development led, for example, to a characterization of associated sets of bounded derivatives ([8]) and of all derivatives ([6]) and many other results in this direction (see surveys $[2,1]$ ) on one side and to a better understanding of the basic difficulty coming from true co-analyticity of the set of differentiable functions on the other side (see [5] and, for related information, [3]). These results imply that a "simple" characterization of derivatives is impossible. This, however, is often easy to see directly. For example, a simple fact that there are a derivative $f$ and a homeomorphism $\varphi$ of $\mathbf{R}$ onto $\mathbf{R}$ such that $\varphi \circ f$ is not a derivative immediately shows that there is no topology $\tau$ on the real line such that the set of derivatives coincides with the set of $\tau$-continuous functions. This has been extended in [7] by showing that one cannot find two topologies $\tau$ and $\sigma$ on $\mathbf{R}$ such that derivatives are characterized as functions continuous from $(\mathbf{R}, \tau)$ to $(\mathbf{R}, \sigma)$. Since continuity is described by the behaviour of inverse images of (some) sets, a natural further extension of these results would be to show that derivatives cannot at all be characterized by prescribing inverse images of sets. More precisely, the problem is whether one can have, for each set $E \subset \mathbf{R}$, a family $\mathscr{F}_{E}$ of subsets of $\mathbf{R}$ such that a function $f$ is a derivative if and only if for each set $E$ the set $f^{-1}(E)$ belongs to $\mathscr{F}_{E}$. One easily sees that not all families of functions can be characterized in this way. An easy example is supplied by the set of bounded functions; an example more related to derivatives is given by the set of Darboux functions.

In the present note we prove that, if $\Delta$ is the set of derivatives, a necessary and sufficient condition for a function $g$ to belong to $\Delta$ is the following:

$$
\forall E \subset \mathbf{R} \exists f \in \Delta: g^{-1}(E)=f^{-1}(E) .
$$

Received by the editors October 4, 1993.

1991 Mathematics Subject Classification. Primary 26A24.

The second author was supported by M.P.I. funds. 
This shows that derivatives can be characterized in the proposed way (with $\left.\mathscr{F}_{E}=\left\{f^{-1}(E): f \in \Delta\right\}\right)$. Even though we do not consider this result as a satisfactory characterization of derivatives (especially because the proof is not constructive), we believe that it opens a new direction in our understanding of their behaviour. In particular, it would be very interesting to know whether one can limit the sets $E$ to some "respectable" class of sets. The only negative result in this direction follows from the above example of a derivative $f$ and a homeomorphism $\varphi$ such that $\varphi \circ f$ is not a derivative, since this easily implies that our statement does not hold if we restrict the sets $E$ to intervals.

We start with the following lemma:

Lemma 1. Let $g$ be a function $\mathbf{R} \rightarrow \mathbf{R}$ and for every subset $E \subset \mathbf{R}$ let $f_{E} \in \Delta$ be such that

$$
g^{-1}(E)=f_{E}^{-1}(E) .
$$

If $g \notin \Delta$ and $h \in \Delta$, then there exist a subset $S$ of $\mathbf{R}$ and a function $f \in \Delta$ such that $\operatorname{card}(S)<c$ (where $c$ denotes the cardinality of $\mathbf{R}), f \neq h$ and

$$
g(x)=f(x)
$$

for every $x \in \mathbf{R} \backslash\left(g^{-1}(S) \cap f^{-1}(S)\right)$.

Proof. Since $\Delta$ contains the set of continuous functions and is contained in the first class of Baire, $\operatorname{card}(\Delta)=c$. (See [4].) Hence the elements of $\Delta$ can be ordered into a transfinite sequence $\left\{f_{\alpha}\right\}$ indexed by ordinals of cardinality less than $c$ such that $f_{0}=h$ and $f_{\alpha} \neq f_{0}$ for $\alpha>0$.

Since $g \notin \Delta$ and $f_{0} \in \Delta$, there is $x_{0} \in \mathbf{R}$ such that $g\left(x_{0}\right) \neq f_{0}\left(x_{0}\right)$. We let $Y_{0}=\left\{g\left(x_{0}\right)\right\}$ and $Z_{0}=\left\{f_{0}\left(x_{0}\right)\right\}$ and, by transfinite induction, we continue to construct increasing sequences of sets $Y_{\beta}$ and $Z_{\beta}$ of power less than $c$ such that $Y_{\beta} \cap Z_{\beta}=\varnothing$ for every $\beta<c$. To this aim, suppose that for every $\beta<\alpha$ the sets $Y_{\beta}$ and $Z_{\beta}$ have been already defined and that $Y_{\beta} \cap Z_{\beta}=\varnothing$ for every $\beta<\alpha$. We let $\widehat{Y}_{\alpha}=\bigcup_{\beta<\alpha} Y_{\beta}$ and $\widehat{Z}_{\alpha}=\bigcup_{\beta<\alpha} Z_{\beta}$ and consider the following three possibilities:

(1) There is $x \in \mathbf{R}$ such that $g(x) \notin \widehat{Z}_{\alpha}, f_{\alpha}(x) \notin \widehat{Y}_{\alpha}$ and $g(x) \neq f_{\alpha}(x)$. We choose one such $x$, say $x_{\alpha}$, and define $Y_{\alpha}=\widehat{Y}_{\alpha} \cup\left\{g\left(x_{\alpha}\right)\right\}$ and $Z_{\alpha}=\widehat{Z}_{\alpha} \cup\left\{f_{\alpha}\left(x_{\alpha}\right)\right\}$. Clearly, our assumptions imply that $Y_{\alpha} \cap Z_{\alpha}=\varnothing$.

(2) The case (1) does not occur and there is $x \in \mathbf{R}$ such that $g(x) \notin \widehat{Y}_{\alpha}$, $f_{\alpha}(x) \notin \widehat{Z}_{\alpha}$ and $g(x) \neq f_{\alpha}(x)$. We choose one such $x$, say $x_{\alpha}$, and define $Y_{\alpha}=\widehat{Y}_{\alpha} \cup\left\{f_{\alpha}\left(x_{\alpha}\right)\right\}$ and $Z_{\alpha}=\widehat{Z}_{\alpha} \cup\left\{g\left(x_{\alpha}\right)\right\}$. Again, our assumptions imply that $Y_{\alpha} \cap Z_{\alpha}=\varnothing$.

(3) Neither (1) nor (2) occurs: In this case the construction stops.

Let us note first that if case (3) occurs for some $\alpha<c$, then the lemma is proved. Indeed, let

$$
S=\widehat{Y}_{\alpha} \cup \widehat{Z}_{\alpha} \text { and } f=f_{\alpha} .
$$

Clearly, $\operatorname{card}(S)<c$ and $f \neq h$ (since $\alpha>0$ ). If $x \in \mathbf{R}$ and $g(x) \neq f_{\alpha}(x)$ we use that case (1) did not occur to infer that $g(x) \in \widehat{Z}_{\alpha}$ or $f_{\alpha}(x) \in \widehat{Y}_{\alpha}$. In the former case we have $g(x) \notin \widehat{Y}_{\alpha}$ (because $\widehat{Y}_{\alpha} \cap \widehat{Z}_{\alpha}=\varnothing$ ) and therefore, since case (2) did not occur, $f_{\alpha}(x) \in \widehat{Z}_{\alpha}$. Hence $g(x) \in \widehat{Z}_{\alpha} \subset S$ and $f_{\alpha}(x) \in \widehat{Z}_{\alpha} \subset S$, which shows that

$$
x \in g^{-1}(S) \cap f^{-1}(S) .
$$


Similarly, in the case when $f_{\alpha}(x) \in \widehat{Y}_{\alpha}$, we have that $f_{\alpha}(x) \notin \widehat{Z}_{\alpha}$ and we deduce that $g(x) \in \widehat{Y}_{\alpha}$. Again, we conclude that

$$
x \in g^{-1}(S) \cap f^{-1}(S) .
$$

Thus every $x \in \mathbf{R}$ for which $g(x) \neq f(x)$ belongs to the set $g^{-1}(S) \cap f^{-1}(S)$, which shows that $g(x)=f(x)$ for $x \in \mathbf{R} \backslash\left(g^{-1}(S) \cap f^{-1}(S)\right)$.

It remains to show that case (3) occurs for some $\alpha<c$. To this aim, suppose on the contrary that the families $\left(Y_{\beta}\right)$ and $\left(Z_{\beta}\right)$ are defined for every $\beta<c$ and put

$$
E=\bigcup_{\beta<c} Y_{\beta} \text { and } F=\bigcup_{\beta<c} Z_{\beta} .
$$

Let $f_{E} \in \Delta$ be such that $g^{-1}(E)=f_{E}^{-1}(E)$. Then $f_{E}=f_{\alpha}$ for some $\alpha<c$. If, for this $\alpha$, case (1) occurred, or if $\alpha=0$, there is $x \in \mathbf{R}$ (namely, the $x_{\alpha}$ chosen in the construction) such that $g(x) \in E$ and $f_{\alpha}(x) \in F$. Since $E \cap F=\varnothing$, this shows that $g^{-1}(E) \neq f_{\alpha}^{-1}(E)$. If case (2) occurred, there is $x \in \mathbf{R}$ such that $g(x) \in F$ and $f_{\alpha}(x) \in E$. Again, we conclude that $g^{-1}(E) \neq f_{\alpha}^{-1}(E)$, which is the desired contradiction.

Lemma 2. Let $g$ be a function $\mathbf{R} \rightarrow \mathbf{R}$ and let $f$ and $h$ be two nonconstant functions from $\Delta$. Let $U$ and $T$ be two subsets of $\mathbf{R}$, both with cardinality less than $c$, such that $g$ equals $f$ in $\mathbf{R} \backslash\left(g^{-1}(U) \cap f^{-1}(U)\right)$ and $g$ equals $h$ in $\mathbf{R} \backslash\left(g^{-1}(T) \cap h^{-1}(T)\right)$. Then $f=h$.

Proof. With $S=U \cup T$ we first observe that $f(x) \in S$ for every

$$
x \in\left[\left(g^{-1}(S) \cap f^{-1}(S)\right) \cup\left(g^{-1}(S) \cap h^{-1}(S)\right)\right] .
$$

Indeed, if $f(x) \notin S$ for some such $x$, we have $x \in\left(g^{-1}(S) \cap h^{-1}(S)\right)$. In particular, $g(x) \in S$, which implies that $g(x) \neq f(x)$. But this can happen only if $x \in\left(g^{-1}(U) \cap f^{-1}(U)\right)$, hence $f(x) \in U \subset S$, which is a contradiction.

Let

$$
Z=\mathbf{R} \backslash\left[\left(g^{-1}(S) \cap f^{-1}(S)\right) \cup\left(g^{-1}(S) \cap h^{-1}(S)\right)\right] .
$$

We note that $Z \neq \varnothing$, since otherwise the previous argument shows that $f(x) \in$ $S$ for every $x \in \mathbf{R}$. But this is impossible, because the range of a nonconstant derivative is an interval and $\operatorname{card}(S)<c$.

Finally, we observe that $f$ and $h$ coincide in $Z$ and that, therefore, the function $t=f-h$ is zero in $Z$. Since $Z \neq \varnothing$, the lemma will be proved once we show that the function $t$ is constant. But to see this, it suffices to observe that $t$ is a derivative with range contained in a set of power less than $c$, namely, in the set

$$
\{0\} \cup\{y-x: x, y \in S\} .
$$

Theorem. Let $g$ be a function $\mathbf{R} \rightarrow \mathbf{R}$. Then $g$ is a derivative if and only if

$$
\forall E \subset \mathbf{R} \exists f \in \Delta: g^{-1}(E)=f^{-1}(E) .
$$

Proof. If $g$ is a derivative, the condition is obviously true. Suppose that the condition is fulfilled. If $g$ is not a derivative, we use Lemma 1 (with an arbitrary $h \in \Delta)$ to find a function $f_{0} \in \Delta$ and a subset $S_{0}$ of $\mathbf{R}$ with $\operatorname{card}\left(S_{0}\right)<c$ and such that

$$
g(x)=f_{0}(x) \quad \text { for } x \in \mathbf{R} \backslash\left(g^{-1}(S) \cap f_{0}^{-1}(S)\right) .
$$


Then we use Lemma 1 once more, this time with $h=f_{0}$, to find a function $f_{1} \in \Delta$ and a subset $S_{1}$ of $\mathbf{R}$ with $\operatorname{card}\left(S_{1}\right)<c$ such that $f_{1} \neq f_{0}$ and

$$
g(x)=f_{1}(x) \text { for } x \in \mathbf{R} \backslash\left(g^{-1}(S) \cap f_{1}^{-1}(S)\right) .
$$

But now the assumptions of Lemma 2 are satisfied with $f=f_{0}, h=f_{1}, U=$ $S_{0}$, and $T=S_{1}$; hence $f_{1}=f_{0}$, which gives a contradiction.

Remark. Let $\mathscr{C}$ be a set of functions $\mathbf{R} \rightarrow \mathbf{R}$. If we attempt to use the above proof with $\Delta$ replaced by $\mathscr{C}$, we note that only the following properties of $\mathscr{C}$ are needed:

(1) $\operatorname{card}(\mathscr{C}) \leq c$.

(2) The range of any nonconstant function from $\mathscr{C}$ has cardinality $c$.

(3) The range of any nonconstant function which can be expressed as a difference of two functions from $\mathscr{C}$ has cardinality $c$.

Hence any set of functions verifying the above conditions can be characterized in the proposed way. In particular, this holds for the set of bounded derivatives.

\section{REFERENCES}

1. A. M. Bruckner, Differentiation of real functions, Lecture Notes in Math., vol. 659, SpringerVerlag, Berlin and New York, 1978.

2. A. M. Bruckner and J. L. Leonard, Derivatives, Amer. Math. Monthly 73 (1966), 24-56.

3. A. S. Kechris and A. Louveau, Descriptive set theory and the structure of sets of uniqueness, Cambridge Univ. Press, Cambridge and New York, 1987.

4. C. Kuratowski, Topology. I, Academic Press, New York and London, 1968.

5. S. Mazurkiewicz, Über die Menge der differenzierbaren Funktionen, Fund. Math. 27 (1936), 244-249.

6. D. Preiss, Level sets of derivatives, Trans. Amer. Math. Soc. 272 (1982), 161-184.

7. M. Tartaglia, Sulla caratterizzazione delle derivate, Pubbl. Dip. Mat. Stat., Napoli, 1988.

8. Z. Zahorski, Sur la première dérivée, Trans. Amer. Math. Soc. 69 (1950), 1-54.

Department of Mathematics, University College London, LoNdon WC1E 6BT, United KINGDOM

E-mail address: dp@math.ucl.ac.uk

Dipartimento di Matematica e Applicazioni, Università di NAPOLI, 80134 Napoli, Italy 